\title{
Specimen Source Site
}

National Cancer Institute

\section{Source}

National Cancer Institute. Specimen Source Site. NCI Thesaurus. Code C159256.

A request to identify the specimen source site. 